\title{
LETTER TO THE EDITOR
}

\section{The discovery of a very massive star in W49}

\author{
S.-W. Wu ${ }^{1, \star \star \star}$, A. Bik ${ }^{1,2}$, Th. Henning ${ }^{1}$, A. Pasquali ${ }^{3}$, W. Brandner ${ }^{1}$, and A. Stolte ${ }^{4}$ \\ 1 Max-Planck-Institut für Astronomie, Königstuhl 17, 69117 Heidelberg, Germany \\ e-mail: shiwei@mpia.de \\ 2 Department of Astronomy, Stockholm University, AlbaNova University Centre, 10691 Stockholm, Sweden \\ 3 Universität Heidelberg, Zentrum für Astronomie, Astronomisches Recheninstitut, Mönchhofstrasse 12-14, 69120 Heidelberg, \\ Germany \\ 4 Argelander Institut für Astronomie, Auf dem Hügel 71, 53121 Bonn, Germany
}

Received 7 May 2014 / Accepted 21 July 2014

ABSTRACT

\begin{abstract}
Context. Very massive stars $\left(M>100 M_{\odot}\right)$ are very rare objects, but have a strong influence on their environment. The formation of this kind of objects is of prime importance in star formation, but observationally still poorly constrained.

Aims. We report on the identification of a very massive star in the central cluster of the star-forming region W49.

Methods. We investigate near-infrared $K$-band spectroscopic observations of W49 from VLT/ISAAC together with $J H K$ images obtained with NTT/SOFI and LBT/LUCI. We derive the spectral type of W49nr1, the brightest star in the dense core of the central cluster of W49.

Results. On the basis of its $K$-band spectrum, W49nr1 is classified as an O2-3.5If* star with a $K$-band absolute magnitude of $-6.27 \pm$ $0.10 \mathrm{mag}$. The effective temperature and bolometric correction are estimated from stars of similar spectral type. After comparison to the Geneva evolutionary models, we find an initial mass between $100 M_{\odot}$ and $180 M_{\odot}$. Varying the extinction law results in a larger initial mass range of $90-250 M_{\odot}$.
\end{abstract}

Key words. stars: formation - stars: massive - supergiants - infrared: stars - techniques: spectroscopic open clusters and associations: individual: W49

\section{Introduction}

Even though very massive stars $\left(M>100 M_{\odot}\right)$ are very rare, they have a strong influence on their environment via powerful winds and ionizing radiation, injecting large quantities of momentum and energy into the surrounding interstellar medium. Their fast evolution and the steep slope of the initial mass function (IMF) imply that one has to study the most massive star-forming regions to identify them.

The formation mechanisms of very massive stars are by no means fully understood (Krumholz 2014). For a long time, it was put in serious doubt whether these very massive stars could actually form at all. Observational evidence was presented suggesting an upper mass limit of $150 M_{\odot}$ (Figer 2005). However, recently Crowther et al. (2010) claimed the existence of very massive stars up to $300 M_{\odot}$, especially in and around young massive clusters, such as NGC 3603, the Arches cluster, and R136 in the Large Magellanic Cloud.

In this Letter we present the discovery of a very massive star in one of the most luminous Galactic H II regions: W49 (Fig. 1). With dozens of OB-type stars in its core, W49 is one of the most

\footnotetext{
* Based on observations made with ESO Telescopes at the La Silla Paranal Observatory under programme IDs 67.C-0514 and 073.D-0837, and on data acquired using the Large Binocular Telescope (LBT).

$\star \star$ The reduced VLT FITS spectrum is only available at the CDS via anonymous ftp to cdsarc. u-strasbg. fr (130.79.128.5) or via http://cdsarc.u-strasbg.fr/viz-bin/qcat?J/A+A/568/L13 $\star \star \star$ International Max Planck Research School for Astronomy and Cosmic Physics at the University of Heidelberg (IMPRS-HD).
}

important Galactic sites for studying the formation and evolution of massive stars in the local universe (Alves \& Homeier 2003; Homeier \& Alves 2005). Given its location in the plane of the Milky Way and a distance of $11.11_{-0.69}^{+0.79} \mathrm{kpc}$ (Zhang et al. 2013), W49 is optically obscured by intervening interstellar dust. This makes an optical identification and spectral classification of the stellar content almost impossible, leaving the near-infrared window (primarily $K$-band) for spectral classification of the highly obscured stars.

Here, we report on the spectroscopic identification of a very massive star in W49, which we hereafter refer to as W49nr1. We first present our near-infrared observations (imaging and spectroscopy) of W49 (Sect. 2). The spectral features and the classification of W49nr1 are described in Sect. 3, where we also derive its stellar parameters like effective temperature $\left(T_{\text {eff }}\right)$, initial mass, and age. Finally, we briefly discuss the implications of our results in Sect. 4 and end with conclusions in Sect. 5.

\section{Observation and data reduction}

A medium-resolution $(R=10000) K$-band spectrum of W49nr1 was obtained with ISAAC mounted on Antu (UT1) of ESO's Very Large Telescope (VLT), Paranal, Chile. Jand $H$-band images were obtained with SOFI at the New Technology Telescope (NTT), La Silla, Chile, and a $K$-band image was acquired with LUCI mounted on the Large Binocular Telescope (LBT), Mount Graham, Arizona. 


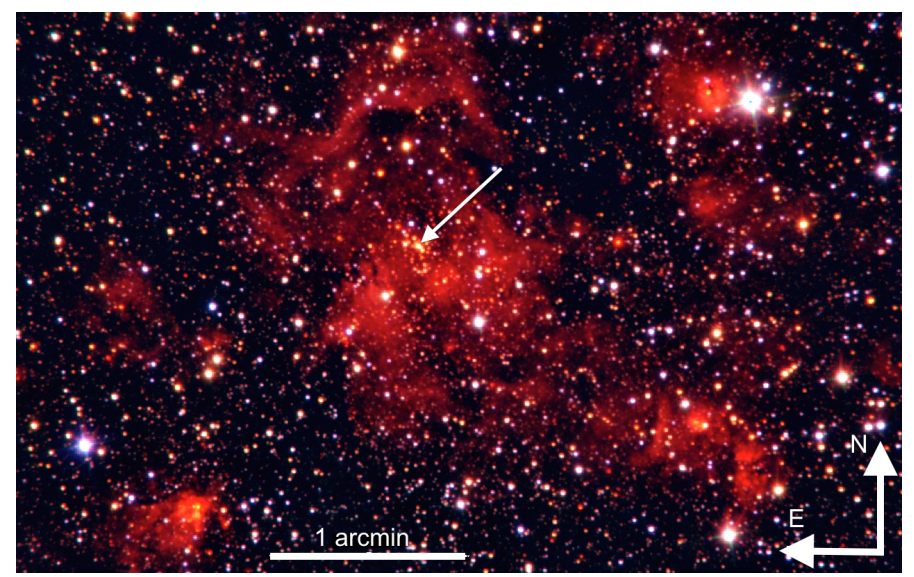

Fig. 1. JHK three colour image of the central area of W49. The massive star W49nr1 is indicated with a white arrow.

Table 1. Observed and derived properties of W49nr1.

\begin{tabular}{lc}
\hline \hline$\alpha(\mathrm{J} 2000)(\mathrm{h} \mathrm{m} \mathrm{s})$ & $19: 10: 17.43$ \\
$\delta(\mathrm{J} 2000)\left({ }^{\prime}{ }^{\prime \prime}\right)$ & $+9: 06: 20.93$ \\
\hline$J(\mathrm{mag})$ & $16.57 \pm 0.18$ \\
$H(\mathrm{mag})$ & $13.47 \pm 0.12$ \\
$K(\mathrm{mag})$ & $11.93 \pm 0.10$ \\
\hline$E W(\mathrm{Br} \gamma)(\AA)$ & $8.2 \pm 1.7$ \\
$E W(\mathrm{He} \mathrm{II})(\AA)$ & $2.4 \pm 0.7$ \\
$E W(\mathrm{~N}$ III $)(\AA)$ & $2.3 \pm 1.0$ \\
$E W(\mathrm{~N})(\AA)$ & $2.6 \pm 0.9$ \\
\hline Spectral type & $\mathrm{O} 2-3.5 \mathrm{If} *$ \\
$T_{\text {eff }}(\mathrm{K})$ & $40000-50000$ \\
$\mathrm{BC}(\mathrm{mag})$ & $-5.2--4.55$ \\
\hline$A_{\mathrm{K}}(\mathrm{mag})$ & $2.9^{a} / 2.6-3.5^{b}$ \\
Initial mass $\left(M_{\odot}\right)$ & $100-180^{a} / 90-250^{b}$ \\
Luminosity $\left(L / L_{\odot}\right)$ & $1.7-3.1 \times 10^{6 a} / 1.2-4.9 \times 10^{6 b}$ \\
\hline
\end{tabular}

Notes. (a) With extinction law of Indebetouw et al. (2005). (b) Considering other extinction laws (see text).

\subsection{Observations}

SOFI $J$ - and $H$-band imaging observations of W49 were performed on 2001 June 7 (PI: Alves) providing a $5^{\prime} \times 5^{\prime}$ field of view with 0.288 pixel $^{-1}$. The data were taken with a DIT (detector integration time) of $6 \mathrm{~s}$ and NDIT (number of integrations) of 5 per saved frame. The number of exposures for $J$ and $H$ band are 20 and 15, respectively, which lead to a total exposure time of $600 \mathrm{~s}(J$ band $)$ and $450 \mathrm{~s}(H$ band $)$. The spatial resolution is $~ 0.5-0.7^{\prime \prime}$.

The LUCI $K$-band data were taken on 2009 September 29 with the N3.75 camera, providing a $5^{\prime} \times 5^{\prime}$ field of view with $0.12 \mathrm{pixel}^{-1}$. The spatial resolution of the $K$ image is $\sim 0.6-0.7^{\prime \prime}$. The observations were taken with a DIT of $2 \mathrm{~s}$ and NDIT of 10. Forty-two frames were observed, resulting in a total exposure time of $840 \mathrm{~s}$. Sky frames were taken at an offset positions centred at $\alpha(2000)=$ $19^{\mathrm{h}} 08^{\mathrm{m}} 35.8^{\mathrm{s}}, \delta(2000)=+08^{\circ} 50^{\prime} 52.7^{\prime \prime}$.

The most luminous star in the central cluster of W49, W49nr1 (Table 1), was observed with ISAAC in the $K$ band on 2004 August 6 (PI: Alves), with three exposures each with a DIT of $300 \mathrm{~s}$. The wavelength range covered by the spectrum is from $2.08 \mu \mathrm{m}$ to $2.20 \mu \mathrm{m}$. The sky frame and science frames were taken with an object-sky-object pattern, and the nodding offset between the two science frames was set to $20^{\prime \prime}$. HR 6572 , an A0V star, was used as the standard star to correct for the telluric features from the atmosphere. It was observed about one hour before the science frames, in the same wavelength range as the science observations and with an integration time of $5 \mathrm{~s}$.

\subsection{Data reduction}

\subsubsection{Imaging}

The $J$ - and $H$-band images were reduced using the ESO SOFI pipeline v1.5.2. and the $K$ data with standard IRAF routines (see also Pasquali et al. 2011; Bik et al. 2014). The images were dark and flat-field corrected. For the $J H$ data, a sky frame was created from the science frames by rejecting the brightest pixels while combining the frames in pixel coordinates. A sky frame for the $K$-band data was created by combining images taken at the offset position and rejecting the three lowest and six highest values.

Photometry on the JHK images was performed with DAOPHOT (Stetson 1987) under IRAF. Stars are detected with daofind with a threshold of $3 \sigma$ above the background. Aperture photometry was performed with phot in a radius of $(1-2) \times$ full width at half maximum (FWHM) of the point spread function (PSF). For each filter a reference PSF model was constructed by combining the PSF of at least 20 objects. The PSF-fitting photometry was performed with allstar - using the PSF model to fit all objects identified with a $3 \sigma$ confidence level over the local background.

The $K$ image of W49 has severe nebular contamination strongly affecting the photometry of the point sources. To reduce the effect of the nebulosity in the $K$ image, we first removed the stars by means of PSF fitting. The residual frame, with all the stars subtracted, was then smoothed with a kernel of 12 pixels, resulting in a frame containing only the smooth nebular emission. This smoothed frame was subtracted from the original frame. After that, we performed PSF photometry on the nebular subtracted image, resulting in a more accurate photometry. The location of W49nr1 is in the centre of a compact cluster and its photometry is affected by crowding from the neighbouring stars. To quantify the effect of the crowding, we performed aperture photometry at the position of W49nr1 on the residuals in the PSF-subtracted frame. This gives an error of 15.8, 6.4, and $8.6 \%$ for $J, H$, and $K$, respectively.

Finally, we cross-matched the obtained catalogues for each filter to identify the sources detected in more than one band. We calibrated the SOFI and LUCI photometry with 2MASS (Skrutskie et al. 2006). The final calibration resulted in errors in the zero points of $0.0063,0.0071$, and $0.0055 \mathrm{mag}$ for the $J, H$ and $K$ band, respectively. We did not find a colour dependence of the derived zero points. The final errors of the $J H K$ photometry, as listed in Table 1, are a combination of the photometry uncertainty, errors in the zero points, and the errors due to crowding.

\subsubsection{Spectroscopy}

The ISAAC observations of W49nr1 were reduced using standard IRAF routines. The wavelength calibration was performed using the Xe and Ar arc frames. After the flat-fielding and wavelength calibration, the sky was removed by subtracting the frames taken at the A and B nodding positions. The spectra were extracted using doslit and the different exposures are combined to one final spectrum. To remove the narrow Bry emission from the diffuse nebular emission surrounding the cluster, the 


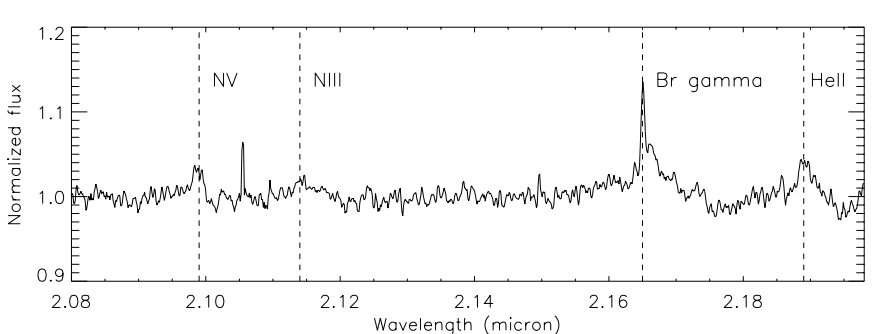

Fig. 2. Normalised $K$-band spectrum of W49nr1 with the emission lines annotated.

background was estimated using a Legendre function, sampling a region close to the star, and subtracted from the spectrum.

The spectrum of the telluric standard star was reduced in the same way as the spectrum of W49nr1. Before correcting the spectrum of W49nr1 with the standard star, the Bry line of the standard star was removed by fitting a Lorentzian profile. The resulting atmospheric transmission spectrum was used to correct the science spectrum for telluric absorption using the IRAF task telluric. The signal-to-noise ratio $(\mathrm{S} / \mathrm{N})$ of the final spectrum is $\sim 90$.

\section{Results}

\subsection{Spectral classification of W49nr1}

The final, normalised $K$-band spectrum of W49nr1 is shown in Fig. 2. The spectrum is dominated by broad emission lines of $\operatorname{Br} \gamma(2.16 \mu \mathrm{m})$, He II $(2.189 \mu \mathrm{m})$, N III $(2.116 \mu \mathrm{m})$, and $\mathrm{NV}(2.10 \mu \mathrm{m})$. The narrow emission component of $\mathrm{Br} \gamma$ is a residual of the nebular subtraction. The He II and $\mathrm{N} \mathrm{V}$ lines are indicative of an early spectral type (Hanson et al. 2005). The broad emission profiles imply an origin in the stellar wind. These properties suggest similarities with the spectral classes O2-3.5If*, O2-3.5If*/WN5-7 ("slash" stars), and WN5-7 stars (Crowther \& Walborn 2011). The sum of the equivalent widths (EWs) of $\mathrm{Br} \gamma$ and He II can be used as a discriminator between these classes. For the WN5-7 stars, the summed EWs is expected to be above $70 \AA$, while O2-3.5If* stars have a total EW between $2 \AA$ and $20 \AA$, with the slash stars lying in between. The total EW of both lines for W49nr1 (Table 1) is $(10.6 \pm 1.8 \AA)$, resulting in a classification of W49nr1 as $\mathrm{O} 2-3.5 \mathrm{If}^{*}$.

\subsection{Hertzsprung-Russell diagram}

Based on the classification of W49nr1 as an O2-3.5If* star, we estimated $T_{\text {eff }}$ between $40000 \mathrm{~K}$ and $50000 \mathrm{~K}$ and the bolometric correction $\left(\mathrm{BC}_{K}\right)$ between $-5.2 \mathrm{mag}$ and -4.55 mag adopting the derived values for an $\mathrm{O}^{2 *}$, O3I, and an O4I star as representative of this class (Crowther \& Walborn 2011). From our $H K$ photometry the absolute $K$-band magnitude was derived to be $-6.27 \pm 0.1 \mathrm{mag}$ by assuming the distance of $11.11 \mathrm{kpc}$ (Zhang et al. 2013), applying the extinction law of Indebetouw et al. (2005) and adopting the intrinsic colour of $(H-K)=$ $-0.1 \mathrm{mag}$ for O3I stars from Martins \& Plez (2006). After applying the $\mathrm{BC}_{K}$, the bolometric magnitude of $\mathrm{W} 49 \mathrm{nr} 1$ was derived to be between -11.47 mag and $-10.82 \mathrm{mag}$, and the corresponding bolometric luminosity between $1.7 \times 10^{6} L_{\odot}$ and $3.1 \times 10^{6} L_{\odot}$.

We plotted the likely parameter space of W49nr1 in the Hertzsprung-Russell-diagram (HRD) as shown in Fig. 3. As $T_{\text {eff }}$ and $\mathrm{BC}_{K}$ are correlated, the likely location of $\mathrm{W} 49 \mathrm{nr} 1$ is a
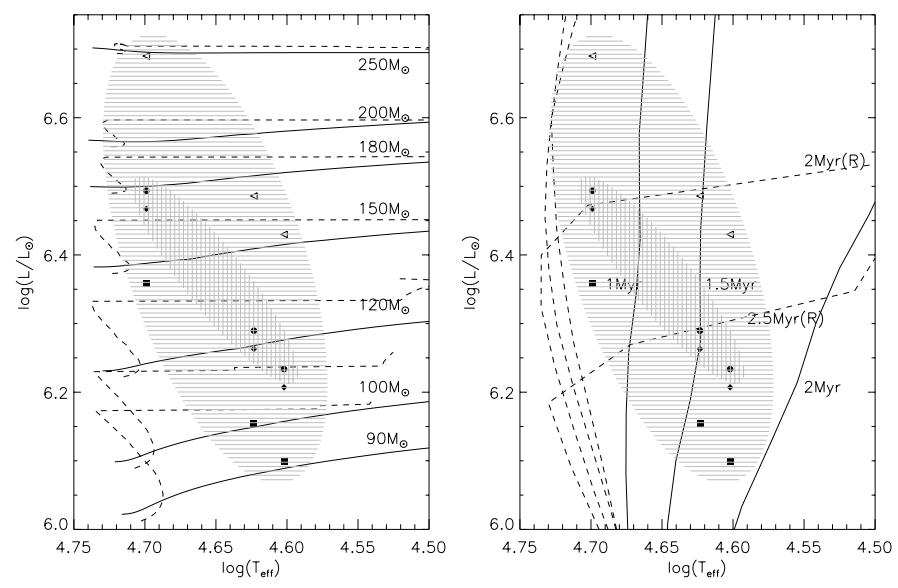

Fig. 3. HRD with the possible location of W49nr1 marked as a vertically hashed area and horizontally hashed, taking into account different extinction laws. The three black filled circles stand for an O2If*, an O3I, and an O4I star, respectively. Left panel: Geneva evolution tracks (Ekström et al. 2012; Yusof et al. 2013) without stellar rotation (solid line) and with rotation (dashed line) for different masses are overplotted. Right panel: main sequence isochrones with different ages, again without stellar rotation (solid line) and with rotation (dashed line).

diagonal ellipse. The possible locations of W49nr1 in the HRD was estimated by calculating the luminosity for the three spectral types in this class (O2If*, O3I, and O4I), using their $T_{\text {eff }}$ and corresponding $\mathrm{BC}_{K}$. To estimate the initial mass and age of W49nr1, the likely parameter space in the HRD was compared with the Geneva theoretical stellar evolution models (Ekström et al. 2012; Yusof et al. 2013), using models with and without stellar rotation.

From the evolutionary tracks, the initial mass of W49nr1 was estimated to be in the range between $\sim 110 M_{\odot}$ and $\sim 180 M_{\odot}$ for models without rotation and between $\sim 100 M_{\odot}$ and $\sim 170 M_{\odot}$ for models with rotation (Fig. 3, left panel, vertically hashed area). While the initial mass estimate for W49nr1 is insensitive to rotation, the isochrones for the models with and without rotation for the same age are very different (Fig. 3, right panel). The position of W49nr1 suggests an upper age limit of 2 Myr after comparison with the non-rotating isochrones, however, considering the models with rotation, an age between $2 \mathrm{Myr}$ and $3 \mathrm{Myr}$ is more likely.

As the extinction towards W49nr1 is high $\left(A_{K}=2.9 \mathrm{mag}\right)$ the choice of the extinction law can have a large effect on the derived luminosity and therefore on its initial mass and age. To select the best fitting extinction laws, we applied a similar analysis to the colour-colour diagram of W49 as Bik et al. (2012) and found that the slopes of the Cardelli et al. (1989) and Román-Zúñiga et al. (2007) laws were not consistent with the observations (Wu et al., in prep.).

The extintion law of Indebetouw et al. (2005) was the best fitting law, but the slopes of Fitzpatrick (1999), Nishiyama et al. (2009) and Rieke \& Lebofsky (1985) are also consistent with the observed colours. Taking into account all four extinction laws, the estimated initial mass range widens to $90-250 M_{\odot}$ (see Fig. 3).

\section{Discussion and future prospectives}

In this letter we report the discovery of a very massive star in the centre of the main cluster in W49. In the following we discuss the uncertainties in the derivation of the stellar parameters 
and the implications for the properties of the central cluster in W49. We end with a suggestion for further characterisation of this object.

\subsection{Stellar paramaters}

Our classification of W49nr1 depends on the empirical relation between the spectral type and the equivalent width of the emission lines as well as the calibration of $K$-band bolometric corrections for early-O stars based upon atmosphere models derived by Crowther \& Walborn (2011). Because very few objects were used in this study, it is hard to predict the uncertainty of this classification and a larger number of stars is needed to make this calibration more reliable.

The evolution of the very massive stars is mostly governed by their stellar wind and mass-loss rate. These input parameters for stellar evolution models add uncertainties to the estimated initial mass and age. As a comparison to the Geneva models we use the relation between the luminosity and the maximum stellar mass for homogeneous hydrogen burners (Gräfener et al. 2011), resulting in a present-day mass estimate of 110-175 $M_{\odot}$ (and 95-250 $M_{\odot}$ when taking into account all four extinction laws as discussed in Sect. 3.2).

The stellar rotation only plays an important part in estimating the age of W49nr1 from the HRD, as the rotational models predict longer time scales for the different evolutionary phases of the massive stars. The $K$-band spectrum is fully dominated by emission lines originating in the stellar wind, hence no estimate of the rotation can be made. High-resolution spectroscopy of possible absorption lines to derive its rotation is crucial to the understanding of the evolutionary status of this extreme star and the cluster. By monitoring the radial velocity of the emission lines, multi-epoch spectroscopy could probe for a possible binary nature.

\subsection{Cluster properties}

The source W49nr1 is located in the centre of the compact central cluster in W49 (Fig. 1), and thus supports the theoretical expectation of rapid dynamical mass segregation (e.g. Allison et al. 2009). Homeier \& Alves (2005) estimate the mass of this cluster as $10^{4} M_{\odot}$. This suggests that W49nr1 is located in an environment quite similar to where other very massive stars located in (Crowther et al. 2010). It adds to the growing number of stars with initial masses at or above $150 M_{\odot}$, suggesting the absence of a strict upper mass limit for massive stars as also suggested by numerical simulations (Kuiper et al. 2010, 2011).

Comparing the cluster mass and the derived stellar mass for W49nr1 to theoretical relations between cluster mass and mass of the most massive star (Weidner et al. 2010) shows that the presence of such a massive star is consistent with a normal IMF. A full study of the upper end of the IMF requires a spectroscopy classification of many more massive stars (Wu et al., in prep.).

\section{Conclusions}

In this Letter we present $J H K_{\mathrm{s}}$ imaging and $K$-band spectroscopy observations of W49nr1, the brightest star in the central cluster of W49. According to classification criteria based on the equivalent widths of $\mathrm{Br} \gamma$ and $\mathrm{He}$ II given by Crowther \& Walborn (2011), W49nr1 is classified as an O2-3.5If* star. We estimate the effective temperature to be between $40000 \mathrm{~K}$ and $50000 \mathrm{~K}$ and the bolometric luminosity between $1.7 \times 10^{6} L_{\odot}$ and $3.1 \times 10^{6} L_{\odot}$. Comparison with the Geneva stellar evolutionary tracks suggests an initial mass range of 100-180 $M_{\odot}$ in the case of a single star, relatively independent of rotational velocity. We study the effect of variations in the extinction law on the stellar parameters, resulting in a large initial mass range of 90-250 $M_{\odot}$. Estimates of the present day mass delivers similar values. The age depends strongly on rotational velocity and can only be constrained to less than 3 Myr. The next step will be a full spectroscopic modelling of the near-infrared spectrum of W49nr1 resulting in stricter constraints on the effective temperature and luminosity. Spectral modelling will allow us to identify possible absorption lines at other wavelengths, suitable for measuring the rotational velocity.

Acknowledgements. We thank the anonymous referee for helpful suggestions that improved the paper significantly. We acknowledge Fabrice Martins and Adrianne Liermann for extensive discussions on the interpretation of the $K$-band spectrum. A.B. acknowledges the hospitality of the Aspen Center for Physics, which is supported by the National Science Foundation Grant No. PHY-1066293. The LBT is an international collaboration among institutions in Germany, Italy, and the United States. LBT Corporation partners are LBT Beteiligungsgesellschaft, Germany, representing the Max Planck Society, the Astrophysical Institute Potsdam, and Heidelberg University; Istituto Nazionale di Astrofisica, Italy; The University of Arizona on behalf of the Arizona University system; The Ohio State University, and The Research Corporation, on behalf of The University of Notre Dame, University of Minnesota and University of Virginia

\section{References}

Allison, R. J., Goodwin, S. P., Parker, R. J., et al. 2009, ApJ, 700, L99 Alves, J., \& Homeier, N. 2003, ApJ, 589, L45 Bik, A., Henning, T., Stolte, A., et al. 2012, ApJ, 744, 87

Bik, A., Stolte, A., Gennaro, M., et al. 2014, A\&A, 561, A12

Cardelli, J. A., Clayton, G. C., \& Mathis, J. S. 1989, ApJ, 345, 245

Crowther, P. A., \& Walborn, N. R. 2011, MNRAS, 416, 1311

Crowther, P. A., Schnurr, O., Hirschi, R., et al. 2010, MNRAS, 408, 731

Ekström, S., Georgy, C., Eggenberger, P., et al. 2012, A\&A, 537, A146

Figer, D. F. 2005, Nature, 434, 192

Fitzpatrick, E. L. 1999, PASP, 111, 63

Gräfener, G., Vink, J. S., de Koter, A., \& Langer, N. 2011, A\&A, 535, A56

Hanson, M. M., Kudritzki, R.-P., Kenworthy, M. A., Puls, J., \& Tokunaga, A. T. 2005, ApJS, 161, 154

Homeier, N. L., \& Alves, J. 2005, A\&A, 430, 481

Indebetouw, R., Mathis, J. S., Babler, B. L., et al. 2005, ApJ, 619, 931

Krumholz, M. R. 2014, to appear in Very Massive Stars in the Local Universe, ed. J. S. Vink [arXiv: 1403.3417]

Kuiper, R., Klahr, H., Beuther, H., \& Henning, T. 2010, ApJ, 722, 1556

Kuiper, R., Klahr, H., Beuther, H., \& Henning, T. 2011, ApJ, 732, 20

Martins, F., \& Plez, B. 2006, A\&A, 457, 637

Nishiyama, S., Tamura, M., Hatano, H., et al. 2009, ApJ, 696, 1407

Pasquali, A., Bik, A., Zibetti, S., et al. 2011, AJ, 141, 132

Rieke, G. H., \& Lebofsky, M. J. 1985, ApJ, 288, 618

Román-Zúñiga, C. G., Lada, C. J., Muench, A., \& Alves, J. F. 2007, ApJ, 664, 357

Skrutskie, M. F., Cutri, R. M., Stiening, R., et al. 2006, AJ, 131, 1163

Stetson, P. B. 1987, PASP, 99, 191

Weidner, C., Kroupa, P., \& Bonnell, I. A. D. 2010, MNRAS, 401, 275

Yusof, N., Hirschi, R., Meynet, G., et al. 2013, MNRAS, 433, 1114

Zhang, B., Reid, M. J., Menten, K. M., et al. 2013, ApJ, 775, 79 